\title{
Acute hemichorea-hemiballism as a sole manifestation of acute thalamic infarct: An unusual occurrence
}

Sir,

Abnormal involuntary movements following stroke are relatively common. They can be hyperkinetic as hemichorea (HC) and hemiballism (HB) or can be hypokinetic as Parkinsonism. Poststroke hyperkinetic movement disorders have an incidence of $0.08 \%{ }^{\left[{ }^{1]}\right.}$ They have been associated with both infarct and cerebral hemorrhage. HC is a unilateral continuous, random, and distally predominant jerking movement that may involve proximal muscles ${ }^{[1]} \mathrm{HB}$ is a unilateral, involuntary, and large amplitude proximal movement. ${ }^{[2]} \mathrm{HC}-\mathrm{HB}$ is an unusual manifestation in thalamic lacunar infarction as compared to more common manifestations such as pure sensory stroke, ataxic hemiparesis, painful ataxic hemiparesis, and hypesthetic ataxic hemiparesis. ${ }^{[3]} \mathrm{We}$ report a patient who presented with sudden onset, HC-HB of right limbs and was found to have acute left thalamic infarct.

A 54-year-old man presented with history of sudden onset involuntary movements in the right arm and leg for 1-day duration. The movements were random, irregular, continuous, becoming more prominent on action than rest, violent. There was no history of similar movement in the left limbs. The patient was known hypertensive for 3 years on medications with no other comorbidities. Family history was not significant. On clinical evaluation, vitals were normal, and he was conscious and oriented. Speech was normal. Motor examination revealed hypotonia of right limbs and hyporeflexia of right limbs. Sensory examination was normal. There was irregular, coarse, continuous, nonsuppressible, violent involuntary movements becoming more prominent on action than rest involving right limbs which were suggestive of right HC-HB [Video 1]. Right plantar was extensor in response. Gait was unsteady due to severe choreoballism. In view of sudden onset of symptoms, unilaterality, and presence of vascular risk factors, cerebrovascular event was considered. Magnetic resonance imaging of the brain showed hyperintensity with restriction on diffusion-weighted imaging in left 


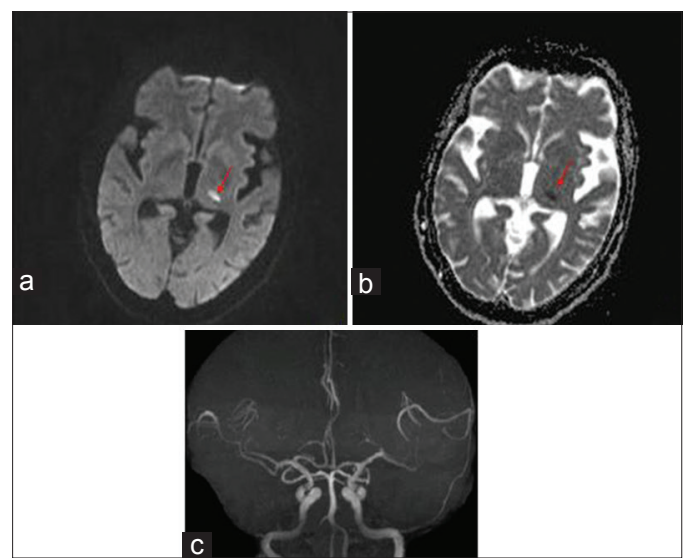

Figure 1: Brain magnetic resonance imaging diffusion-weighted imaging image (a) showing infarct in left lateral thalamus (red arrow); apparent diffusion coefficient image (b) showing corresponding dark signal in left lateral thalamus (red arrow); magnetic resonance angiography (c) is normal

thalamus suggestive of infarct [Figure 1]. Magnetic resonance angiography of extra- and intra-cranial arteries did not reveal any significant abnormality. His complete hemogram, renal, liver, and thyroid function tests were normal. Fasting and postprandial blood glucose were normal. Carotid and vertebral artery Doppler and two-dimensional echocardiography were normal. Serological testing for human immunodeficiency virus, hepatitis B surface antigen, and venereal disease research laboratory was negative. Serum antinuclear antibodies were negative. He was started on aspirin (150 mg daily), atorvastatin (20 mg daily), and carbamazepine (400 mg daily). There was improvement in his involuntary movements and was ambulant at 1-month follow-up [Video 2].

$\mathrm{HC}$ is the most common movement disorder following stroke. Vascular HC is typically associated with ischemic or hemorrhagic lesions of the basal ganglia. The incidence of $\mathrm{HC}-\mathrm{HB}$ in acute stroke ranges between $0.4 \%$ and $0.54 \%$, with a prevalence of $1 \% .{ }^{[4]}$ A study from a tertiary referral center revealed that cerebrovascular disease was the most common cause of nongenetic chorea ${ }^{[5]}$ Lesions of the contralateral striatum are more commonly associated with HC-HB apart from the subthalamic nucleus (STN) ${ }^{[6]}$ In a retrospective study by Pareés et al., on poststroke $\mathrm{HC}$, the most frequent site was in the lentiform nucleus, followed by cortical, thalamic, and subthalamic regions. ${ }^{[7]}$ Vascular territories implicated in HC-HB include lateral lenticulostriate branches of middle cerebral artery; thalamoperforators, thalamogeniculate, and choroidal arteries of posterior cerebral artery; and recurrent artery of Huebner of anterior cerebral artery. ${ }^{[1]}$ Lacunar infarcts constitute about two-third of patients with poststroke hyperkinetic movement disorders; whereas large vessel disease, cardioembolism, and hemorrhage constitute the remainder. ${ }^{[1]}$

Typically, the lesions causing HC-HB occur in the corpus striatum and STN. Thalamic lacunar infarction usually presents as pure sensory stroke, ataxic hemiparesis, and hypesthetic ataxic hemiparesis. HC-HB is the rarest neurological symptom of thalamic infarction. D'Olhaberriague et al. in their study on the 22 patients with movement disorders in ischemic stroke reported 2 patients with HC-HB secondary to thalamic lacunar infarction. ${ }^{[8]}$ Takahashi et al. reported an elderly man with pure $\mathrm{HC}$ due to acute lacunar thalamic infarct. Our patient also had pure HC-HB in association with acute thalamic infarct. ${ }^{[9]}$

The pathophysiological mechanism of HC-HB that is described due to lesion of striatum and STN is as follow: Lesions of the contralateral striatum interrupt inhibitory gamma-aminobutyric acid pathways to the globus pallidus externa (GPe) resulting in increased GPe neuronal activity. The increased inhibitory GPe neuronal activity causes greater inhibition of neurons within the STN. Increased inhibition of the STN leads to a loss of excitatory control over the globus pallidus interna (GPi) causing disinhibition of the motor thalamus. The deficient GPi inhibitory input to the motor component of the thalamus results in excessive thalamocortical motor movement causing hyperkinetic movements. ${ }^{[6]}$

The pathophysiological mechanism of HC-HB described in the thalamic lesion is the disruption of the various thalamic connecting fibers from the STN, globus pallidus, posterior limb of internal capsule, and cerebellum, leading to the crucial derangement in the basal ganglia-cortical circuit. ${ }^{[9]}$ Takahashi et al. did single-photon emission computed tomography study on their patient and found contralateral thalamic hypoperfusion and striatal hyperperfusion. Striatal hyperperfusion suggested increase in the striatal neuronal inhibitory activity on $\mathrm{GPi}$, thus, causing disinhibition of the thalamic neurons. ${ }^{[9]}$

There are very few reports of the occurrence of HC-HB following thalamic lacunar infarction. The present case reports the relatively rare occurrence of $\mathrm{HC}-\mathrm{HB}$ following thalamic lacunar infarction. The pathophysiology of chorea and location of the lesion producing it are not fully understood. Understanding the pathophysiological mechanism responsible for the HC-HB following thalamic infarction needs further research.

\section{Financial support and sponsorship}

Nil. 


\section{Conflicts of interest}

There are no conflicts of interest.

Rohan R. Mahale, Anish Mehta, Aju Abraham John, Kiran Buddaraju, Abhinandan K. Shankar, Rangasetty Srinivasa

Department of Neurology, MS Ramaiah Medical College and Hospital, Bengaluru, Karnataka, India

Address for correspondence: Dr. Rohan R. Mahale, Department of Neurology, MS Ramaiah Medical College and Hospital, Bengaluru - 560 054, Karnataka, India. E-mail: rohanmahale83@gmail.com

\section{References}

1. Handley A, Medcalf P, Hellier K, Dutta D. Movement disorders after stroke. Age Ageing 2009;38:260-6.

2. Dewey RB Jr, Jankovic J. Hemiballism-hemichorea. Clinical and pharmacologic findings in 21 patients. Arch Neurol 1989;46:862-7.

3. Kim JW, Kim SH, Cha JK. Pseudochoreoathetosis in four patients with hypesthetic ataxic hemiparesis in a thalamic lesion. J Neurol 1999;246:1075-9.

4. Ghika-Schmid F, Ghika J, Regli F, Bogousslavsky J. Hyperkinetic movement disorders during and after acute stroke: The Lausanne Stroke Registry. J Neurol Sci 1997;146:109-16.

5. Piccolo I, Defanti CA, Soliveri P, Volontè MA, Cislaghi G, Girotti F. Cause and course in a series of patients with sporadic chorea. J Neurol 2003;250:429-35.

6. Chung SJ, Im JH, Lee MC, Kim JS. Hemichorea after stroke:
Clinical-radiological correlation. J Neurol 2004;251:725-9.

7. Pareés I, Hernández-Vara J, Álvarez-Sabín J. Post-stroke hemichorea: Observation-based study of 15 cases. Rev Neurol 2010;51:460-4.

8. D'Olhaberriague L, Arboix A, Martí-Vilalta JL, Moral A, Massons J. Movement disorders in ischemic stroke: Clinical study of 22 patients. Eur J Neurol 1995;2:553-7.

9. Takahashi T, Kanamori H, Shigehara R, Takahashi SN, Tamura M, Takasu T, et al. Pure hemi-chorea resulting from an acute phase of contralateral thalamic lacunar infarction: A case report. Case Rep Neurol 2012;4:194-201

This is an open access article distributed under the terms of the Creative Commons Attribution-NonCommercial-ShareAlike 3.0 License, which allows others to remix, tweak, and build upon the work non-commercially, as long as the author is credited and the new creations are licensed under the identical terms.

Videos Available on: www.ruralneuropractice.com

\begin{tabular}{|l|l|}
\hline \multicolumn{2}{|c|}{ Access this article online } \\
\hline Quick Response Code: & Website: \\
\hline & www.ruralneuropractice.com \\
\cline { 2 - 2 } & \\
\hline
\end{tabular}

How to cite this article: Mahale RR, Mehta A, John AA, Buddaraju $\mathrm{K}$ Shankar AK, Srinivasa R. Acute hemichorea-hemiballism as a sole manifestation of acute thalamic infarct: An unusual occurrence. J Neurosci Rural Pract 2016;7:478-80. 